\title{
Tirocinio e sviluppo professionale degli educatori nella prospettiva della Student Voice
}

\author{
di Giovanni Bonaiuti ${ }^{\wedge}$ Ludovica Fanni ${ }^{\circ *}$
}

\section{Riassunto}

Il seguente articolo intende dar spazio all'esperienza del tirocinio curricolare nella prospettiva della Student Voice, sottolineandone l'aspetto altamente formativo. Grazie alla somministrazione di questionari e interviste tra gli studenti del Corso di Laurea in L-19, tra i fattori emersi, spicca il valore che $\mathrm{i}$ futuri educatori conferiscono a tutti i momenti del tirocinio, dall'orientamento alla relazione conclusiva. Tra i vari periodi di cui si compone questa esperienza gli studenti avvertono l'esigenza di una maggiore presenza dei momenti precedenti al suo avvio. Nello specifico l'orientamento e la preparazione sono i momenti dei quali gli studenti sentono una necessità più forte, nata dal poco spazio a questi dedicato e dalla volontà di intraprendere il percorso del tirocinio con una maggiore consapevolezza di ciò che questo evento rappresenta per il futuro lavorativo e quindi avere le basi utili alla comprensione di ciò che sarà il loro agire professionale all'interno di un contesto specifico.

Parole chiave: Tirocinio formativo, Student Voice, Università, educazione, orientamento e preparazione al lavoro, consapevolezza professionale, riflessività

\section{Abstract. Internship and Professional Development for Educators in the Student Voice Perspective}

This paper reflects on viable criteria to ameliorate the experience of curricular academic internship from the perspective of the "Student Voice". Such an approach takes students' opinions into account with an aim to enhance or change education policies. This aspect is particularly relevant for

\footnotetext{
^Università di Cagliari. Corresponding author: g.bonaiuti@unica.it.

${ }^{\circ}$ Università di Cagliari.

* Il lavoro è stato concepito collaborativamente. Giovanni Bonaiuti è autore dei paragrafi: Introduzione, La centralità dell'orientamento, e Il questionario. Ludovica Fanni dei paragrafi: Il tirocinio come esperienza professionalizzante, Contesto della ricerca. Strumenti e metodi, Le interviste, e Considerazioni conclusive.
}

Educational Reflective Practices (ISSNe 2279-9605), 2/2021

Doi: 10.3280/erp2-2021oa12120 
the degree courses in the Pedagogy area, increasingly engaged in the difficult search for a balance between a broad, flexible, and multi-faceted cultural preparation and professional training. Much research has focused on the importance of internship experiences to develop students' professionalization. Our research presents the results obtained from the administration of a questionnaire and focused interviews. The results that emerged led to the Board of Studies' decision to implement several corrections to some critical aspects of the experience. Among the needs reported by the students feature orientation and initial preparation: aspects on which the course has undertaken corrective measures.

Keywords: Internship, Student Voice, University, education, orientation and job training, professional awareness, responsiveness

First submission: 05/05/2021, accepted: 17/05/2021

Available online: 02/07/2021

\section{Introduzione}

Il presente contributo intende offrire una riflessione sul tema dei criteri attuativi dei tirocini formativi curricolari in ambito universitario, aspetto che assume particolare rilevanza in particolare nei corsi dell'area pedagogica sempre più impegnati nella difficile ricerca di un equilibrio tra una preparazione culturale ampia, flessibile e plurale e una formazione professionalizzante mirata a garantire la capacità di operare in una molteplicità di campi educativi che vanno dalla primissima infanzia alla vecchiaia. In particolare, si desidera fornire l'esperienza sviluppata presso l'Università degli Studi di Cagliari nell'ambito del corso di Studi L-19, Scienze dell'educazione e della formazione, che a partire dal recepimento del D.M. 378/2018 che definisce $\mathrm{i}$ crediti minimi per il conseguimento del titolo di educatore dei servizi educativi per l'infanzia, ha dovuto affrontare la complessa gestione di due diversi tirocini formativi: uno nelle strutture formative dell'infanzia/nido e uno nelle altre strutture connesse all'esercizio della professione dell'educatore professionale socio-pedagogico (L. 205/2017). Il corso con una modifica ordinamentale che lo ha portato dall'anno accademico 2019/20 ad ampliare la propria offerta formativa con insegnamenti e laboratori, sulla carta sicuramente rispondenti alle finalità formative, culturali e professionali previste dalla normativa - per altro da tempo sollecitate dai rappresentanti delle parti interessate rappresentate nel suo Comitato di indirizzo - ha sentito l'esigenza di comprendere se il nuovo assetto risultasse effettivamente idoneo a favorire il raggiungimento di una idonea preparazione degli studenti. Per rispondere 
a tali interrogativi, sfruttando anche le opportunità offerte dalla partecipazione al Progetto POT "Percorsi di orientamento e tutorato per promuovere il successo universitario e professionale" , sono state intraprese una molteplicità di azioni tese sia a facilitare l'orientamento e l'accompagnamento degli studenti in particolare ai percorsi professionalizzazione, sia al monitoraggio degli esiti. La prospettiva che ha ispirato questo secondo aspetto, e dunque il presente lavoro, è quella della "Student Voice" (Grion \& Cook-Sather, 2013) che, come noto, reputa centrale il considerare il punto di vista degli studenti quale prospettiva di elezione per il cambiamento migliorativo nell'offerta e nelle attività promosse dagli atenei. La voce degli studenti non solo ha il diritto di essere ascoltata e rispettata perché consente di comprendere le necessità e i bisogni che devono essere al centro del processo di apprendimento al fine di evitare le conseguenze che un insegnamento e esperienze poco funzionali possono portare con sé, ma anche perché questo consente di sfruttare il potenziale trasformativo presente nel coinvolgimento di studenti e docenti nel raggiungimento di fini emancipatori e nella trasparente condivisione di obiettivi e valori (Fielding, 2001; Cook-Sather, 2002). Rendere autorevole la voce degli studenti significa rivisitare i modi tradizionali di concepire e fare università arrivando, in particolare, a condividere l'idea di un'offerta formativa efficace per loro che si apprestano a diventare i professionisti dell'educazione (Fabbri, 2018). Ai fini del nostro lavoro, lo sguardo degli studenti sulle loro esperienze di tirocinio assunte attraverso la rielaborazione da parte della Commissione tirocini a cui hanno fattivamente contribuito i rappresentanti degli studenti eletti nel Consiglio di corso ed ex studenti attivi nel gruppo di lavoro che ha portato avanti le iniziative del progetto POT, ha consentito di focalizzare e affrontare alcune problematiche suscettibili di impedire la piena valorizzazione dei tirocini e dunque di dispiegarne il potenziale trasformativo. Mettere gli studenti al centro, coinvolgerli attivamente nei processi di riflessione sul miglioramento e sul cambiamento significa, come suggerito dall'ampia letteratura sulla Student Voice (Di Vita, 2017), accogliere l'idea che lo studente non sia solo il "destinatario" della formazione, ma un collaboratore essenziale sia nella valutazione delle decisioni politiche, sia nell'orientamento dell'azione formativa e nella messa a punto delle modalità di attuazione del curricolo.

\footnotetext{
${ }^{1}$ Il progetto, il cui capofila è 1'Università di Siena, ha beneficiato del finanziamento ministeriale relativo ai fondi POT 2017/2018 (Decreto MIUR 359 del 4/3/2019), ed ha visto la partecipazione di undici atenei che hanno permesso la condivisione di esperienze e rappresentato lo spunto attuativo delle azioni da cui origina il presente lavoro.
} 


\section{Il tirocinio come esperienza professionalizzante}

Ciò che ci si aspetta dai sistemi formativi è che preparino studenti capaci di affrontare le sfide e le incertezze di una società liquida, in cui - come sostiene Bauman (2008) - le relazioni, i legami e le dinamiche, comprese quelle lavorative, sono sempre più fragili e mutevoli. La sfida diventa particolarmente impegnativa per i percorsi chiamati a formare professionisti dell'educazione, ovvero figure professionali più "liquide" di altre (Spuznar \& Renda, 1998), dal momento che non solo dovranno far fronte ai problemi di adattamento ad esempio a situazioni ed esperienze lavorative precarie, complesse e frammentarie, ma dovranno allo stesso tempo riuscire a sviluppare la capacità di agire con competenza in situazioni costitutivamente incerte, sfuggenti, costantemente in via di definizione in cui il loro ruolo dovrà continuamente reinventarsi per fornire ai soggetti e nelle situazioni in cui opereranno i servizi e il supporto attesi. In questo senso si tratta di una formazione che paradossalmente scommetta sulla capacità di trasformare la debolezza e l'incertezza in elementi salutari perché collocati nell'ininterrotta ricerca sul senso dell'agire educativo come tensione continua all'apprendimento e alla ri-progettazione dei propri interventi (Tramma, 2008). Si tratta dunque di intervenire affinché alla preparazione culturale, e dunque ai saperi di ordine pedagogico, psicologico, filosofico e sociologico che dovranno sostenere l'azione, si uniscano occasioni di sviluppo di quelle capacità trasversali - osservative, riflessive, organizzative, progettuali, relazionali - necessarie ad operare in maniera critica e consapevole nei contesti dell'intervento educativo (Deiana, 2019). Sul fronte dello sviluppo di queste capacità professionali un ruolo preminente, nella formazione universitaria, è svolto dai laboratori e dai tirocini. Sono infatti questi i luoghi dell'incontro anticipato di quella che sarà la vita professionale, il momento dove lo studente può sperimentare e analizzare, in situazione protetta, la complessità dell'agire, quindi luoghi dell'azione riflessiva e della partecipazione attenta alle pratiche sebbene in modalità contenuta, controllata, discreta ma al tempo stesso consapevole e vigile. L'importanza del ruolo svolto dal tirocinio nella formazione universitaria per lo sviluppo di competenze professionali trasversali e specifiche, in particolare, è ormai riconosciuta a livello internazionale (Del Gobbo, Frison, \& Pellegrini, 2021) e lo è in maniera particolare per le professioni educative (Spuznar \& Renda, 1998). Il tirocinio come setting privilegiato per apprendere dalla pratica, attraverso il fare e la riflessione sulle sue conseguenze all'interno di un quadro temporale predefinito e di uno spazio d'azione circoscritto da quelli che sono gli obiettivi predefiniti in fase progettuale e sotto il costante accompagnamento e supervisione di un esperto presenta un duplice valore: formativo e orientativo (Bastianoni \& Spaggiari, 
2016). Formativo perché arricchisce le conoscenze, le competenze e le abilità degli studenti, orientativo perché garantisce attraverso l'esperienza che solo la pratica può fornire l'opportunità di sperimentarsi nei diversi ambiti così da definire e comprendere meglio, anche sul piano vocazionale, le traiettorie del proprio sviluppo professionale. È solo nel corso di questa esperienza, infatti, che lo studente ha la possibilità di individuare i propri limiti e le proprie potenzialità, oltre che di valutare l'adeguatezza e l'utilità delle preesistenti prefigurazioni: dalle idealità ai pregiudizi, dall'immaginario agli stereotipi che magari erano alla base della scelta del percorso di studi e che hanno un ruolo rilevante nel dare forma ai pensieri e orientare le azioni (Bastianoni \& Spaggiari, 2016). Solo attraverso l'agire in situazione e la partecipazione, sebbene periferica, ma comunque legittimata, alle diverse comunità di pratica (Lave \& Wenger, 2007; Wenger, 2006) che compongono il variegato mondo dell'educazione e della formazione «il tirocinante può appropriarsi degli specifici "attrezzi" della professione (tecniche, strumenti e linguaggi), di sovraordinate capacità progettuali, riflessive e valutative e, contestualmente, contribuire alla costruzione del sapere condiviso all'interno della comunità professionale» (Spuznar \& Renda, 1998). L'esperienza diretta in un contesto professionale non è però solo l'occasione per integrare le conoscenze teoriche e metodologiche acquisite con lo studio, né solo la possibilità di agevolare la transizione al mondo del lavoro. Rappresenta piuttosto, e in primo luogo, il momento in cui si impara ad osservare e riflettere sull'azione nel momento stesso in cui questa avviene. Questo tipo di riflessione, la sola capace di permettere di governare la complessità e andare oltre alle conoscenze tecnico-razionale sempre inadeguate ad affrontare le incertezze e gli imprevisti della realtà richiede un atteggiamento di apertura al dubbio, alla possibilità, all'innovazione che diversamente rimarrebbero precluse (Schön, 1993; Mezirow, 2003). La circolarità necessaria tra azione e riflessione, come già suggeriva Dewey (1961) consente agli individui di emanciparsi da attività meramente impulsive $\mathrm{o}$ abitudinarie diventando un'opportunità per sviluppare piani d'azione coerenti con i propositi, di agire in maniera deliberata e intenzionale per raggiungere i risultati desiderati senza per questo imbrigliare l'azione in stereotipie, atteggiamenti e pensieri limitati e limitanti. Valorizzare il tirocinio, renderlo esperienza capace di ampliarsi fino a diventare una sorta di "palestra per la riflessività" richiede, riprendendo il pensiero della Mortari (2009), un articolato lavoro in diverse direzioni e in diversi momenti. Significa, ad esempio, imparare a riflettere "in" azione ovvero a sviluppare l'attitudine a un'attenzione vigile rispetto a quanto accade nel contesto nel momento stesso in cui l'azione si svolge. Significa però, e anche, riflettere "sull'azione" dopo che questa si è svolta al fine di ricostruire il processo, gli eventi che hanno deter- 
minato le scelte e i pensieri che hanno suggerito le direzioni riuscendo, al contempo, ad interpretare le motivazioni e le conseguenze dell'agire. Significa, infine, abituarsi a riflettere sulle alternative e sui piani di azione possibili e saperlo fare in maniera anticipata, sia prima dell'azione, sia al suo termine in vista di nuove e possibili prosecuzioni. Da questo tipo di riflessione anticipatrice discende la capacità di ipotizzare scenari, pianificare, progettare e mettere a punto criteri per valutare esiti e conseguenze.

\section{La centralità dell'orientamento}

È fondamentale che il sistema formativo comprenda le sfide e i cambiamenti del mondo del lavoro educativo per riuscire ad affiancare lo studente nel passaggio verso il mondo del lavoro (Bufalino, 2000), ma è altrettanto necessario investire sull'accompagnamento alla maturazione di un pensiero riflessivo capace di rendere permanente quell'atteggiamento attento, critico, aperto al ripensamento e alla significazione che permetterà nella futura vita professionale di affrontare, nonostante l'esperienza, ogni situazione come nuova, sfidante, incerta, occasione inedita per continuare a crescere e imparare. Determinante in questo percorso è la figura del tutor che rende possibile la realizzazione di azioni di orientamento, supporto e monitoraggio qualificate, che possano costruire itinerari competitivi di formazione alla pratica lavorativa. Ciò rende questa figura strategica per la qualificazione del tirocinio formativo come «leva di placement» (De Canale, 2015). È importante tenere conto del fatto che il tirocinio curricolare per molti studenti rappresenta il primo ingresso all'interno del mondo del lavoro. Attraverso esso il giovane inizia ad apprendere cosa significhi concretamente vivere parallelamente all'interno di una pluralità di contesti. Il tirocinio, dunque, diventa prezioso strumento che permette allo studente di fare esperienza dall'esperienza, processo circolare che intreccia conoscenze e abilità, pensiero e azione, teoria e pratica, interno ed esterno, formazione formale con apprendimenti informali. Si tratta dunque di una esperienza che non può prescindere dal rapporto tra università e strutture ospitanti, tra tutor interni all'ateneo e tutor della struttura ospitante. È soprattutto questa seconda figura, tipicamente un educatore o un pedagogista, quindi un collega esperto, ad assumere un particolare significato nel rendere il tirocinio una esperienza orientata alla costruzione di una propria professionalità (Bastianoni \& Spaggiari, 2015). A partire dal primo ricevimento con il tutor dell'ente accogliente, che inaugura l'incontro con il contesto operativo, prende corpo la duplice natura di un'esperienza la cui funzione orientativa è inscindibilmente caratterizzata da una valenza formativa nel corso della quale lo studente sperimenta in prima persona l'importanza dell'accoglienza e il valore della relazione nella dimensione dell'ascolto e della cura. 
Emerge quindi l'importanza del nodo triadico che vede lo studente al centro di un rapporto che da un lato ha il tutor accogliente dell'ente e dall'altro l'università mediante le figure dei tutor universitari. Da questo intreccio dipende buona parte della qualità dell'esperienza. Se l'università non conosce, non dialoga, non si relaziona con le realtà educative il rischio è quello di esperienze separate dove quando va bene si determinano percorsi su binari paralleli che finiscono per non incontrarsi mai e quando va male si hanno esperienze contraddittorie, antinomiche in cui la teoria rischia di risultare incompatibile o inconciliabile con la pratica.

Solo se l'università conosce le realtà alle quali affida i propri studenti e solo se queste hanno occasioni di contatto e condivisione con l'università si assiste ad un intreccio coerente capace di fornire sostegno allo studente e garantirgli la possibilità di "imparare ad imparare" dal contesto in cui si trova ad agire (Santoro, 2015).

\section{Contesto della ricerca. Strumenti e metodi}

Allo scopo di migliorare le esperienze di tirocinio dei propri studenti, il corso di studi di Scienze dell'educazione e della formazione dell'Università di Cagliari, attraverso la sua Commissione tirocini, grazie anche alle occasioni di incontro e di dialogo con i colleghi dei corsi di studio delle università italiane aderenti al progetto POT "Percorsi di orientamento e tutorato per promuovere il successo universitario e professionale", ha intrapreso una serie di azioni sinergiche volte a rafforzare le occasioni di incontro e contatto tra enti operanti nell'ambito dell'educazione e della formazione e università, ad esempio promuovendo seminari di studio e incontri volti a facilitare la conoscenza delle realtà territoriali e dunque tesi a irrobustire la preparazione iniziale degli studenti in vista dell'esperienza di tirocinio. In vista del miglioramento del supporto allo svolgimento del primo tirocinio formativo, momento reputato critico da parte dei docenti tutor del corso di studi, si è ritenuto opportuno indagare meglio quali fossero state le reali percezioni degli studenti rispetto a questa esperienza professionalizzante.

A questo scopo sono stati individuati due strumenti principali di rilevazione: il questionario (allegato 1) e l'intervista in profondità. Il primo strumento, composto da ventidue domande, prevalentemente a risposta multipla su una scala a cinque punti ("molto/per nulla") oltre ad alcune opzioni di risposta chiusa dicotomica ("sì/no") e spazi di risposta a commento aperto per alcune domande, è stato somministrato a partire dal mese di maggio 2020 a tutte le studentesse e gli studenti del Corso di studi in Scienze dell'educazione e della formazione dell'Università di Cagliari al momento della conclusione del loro tirocinio. Il secondo invece si riferisce alla realizzazione di interviste a domanda aperta dalle quali si è scelto di estrapolare delle porzioni 
di informazioni sulla base di ciò che è emerso dalle stesse. Le sezioni del questionario erano volte a indagare, comprendere e valutare aspetti quali:

- l'iniziale consapevolezza da parte degli studenti/sse degli obiettivi del tirocinio formativo;

- la completa o parziale corrispondenza tra obiettivi attesi e obiettivi raggiunti al termine del tirocinio;

- l'adeguatezza del percorso formativo del corso di studi in riferimento al tirocinio;

- le conoscenze, i saperi, le abilità e le competenze specifiche del profilo professionale acquisite, approfondite e migliorate durante la formazione universitaria e l'esperienza di tirocinio;

- il canale e i criteri di scelta dell'ente/struttura;

- le attività di ricerca, documentazione e approfondimento dell'ambito e del contesto educativo in vista del tirocinio, in maniera individuale e volontaria da parte del/lla tirocinante;

- la presenza e la qualità dei servizi e delle attività di accompagnamentoorientamento formativo universitario, di assistenza amministrativo-burocratica, di supporto da parte dei tutor universitari ed aziendali;

- il rapporto e la corrispondenza tra tirocinio e inserimento lavorativo;

- la valutazione e il giudizio complessivo dell'esperienza di tirocinio.

Attraverso l'intervista invece si è cercato di andare più a fondo dell'esperienza da un punto di vista interiore, legato alle emozioni che hanno guidato gli studenti. Per approfondire al meglio questi aspetti si è pensato a delle interviste individuali, condotte a distanza tramite la piattaforma Teams. Gli elementi esplorati maggiormente durante questi incontri sono stati quelli connessi:

- alla consapevolezza legata alla scelta del contesto in cui si è svolto il tirocinio;

- alla prima impressione;

- alle prime difficoltà;

- alle aspettative poste sull'esperienza, confrontate alle attività poi realmente svolte, ed i sentimenti ad esse collegate.

\section{Il questionario}

Tra il maggio 2020 e il marzo 2021, periodo per altro caratterizzato dalle difficoltà determinate dall'emergenza sanitaria causata dall'epidemia da COVID-19, hanno svolto e compilato il questionario 131 studenti, 122 donne (93\%) e 9 uomini $(7 \%)^{2}$. Il primo dato da cui partire per l'analisi dell'esperienza è quello relativo alla

\footnotetext{
${ }^{2} \mathrm{Si}$ precisa che il CdS è a numero chiuso programmato a livello locale e immatricola 230 studenti all'anno.
} 
domanda "Nel complesso, come giudica la sua esperienza di tirocinio?" a cui hanno risposto "molto positiva" o "abbastanza positiva" 119 studenti (il 91\%), mentre solo 5 studenti hanno indicato "abbastanza negativa" (meno del 4\%).

Anche dagli altri dati si evince che in generale gli/le studenti/sse siano generalmente soddisfatti del percorso di formazione e della relativa esperienza di tirocinio compiuta. Emerge inoltre come buona parte di loro abbia ritenuto necessario e importante documentarsi, ricercare e approfondire la propria conoscenza in merito ai saperi, al contesto, al ruolo e alle pratiche educative da attuare nella specifica sede ed ambito di tirocinio prescelto in modo autonomo. Allo specifico quesito che richiedeva questo (domanda 16) il 64,89\% degli studenti afferma di essersi documentato e aver riflettuto sul ruolo professionale, il $32,82 \%$ di averlo fatto solo in parte e solo il $2,29 \%$ dichiara di non averlo fatto.

Ciò nonostante, la lettura attenta delle risposte, mette in luce anche alcune criticità relativamente alle quali il Corso ha già preso importanti decisioni $\mathrm{e}$ definito i primi correttivi. Innanzi tutto, benché una buona parte delle risposte indichi adeguato il percorso formativo del corso di studi per affrontare l'esperienza di tirocinio (domanda 6.3), ci sono un $20 \%$ di studenti che hanno espresso un parere contrario (vedi grafico 1) lasciando intendere la presenza di qualche criticità nel rapporto tra teoria e pratica o, più plausibilmente, nell'adeguato accompagnamento al trasferimento dei saperi.

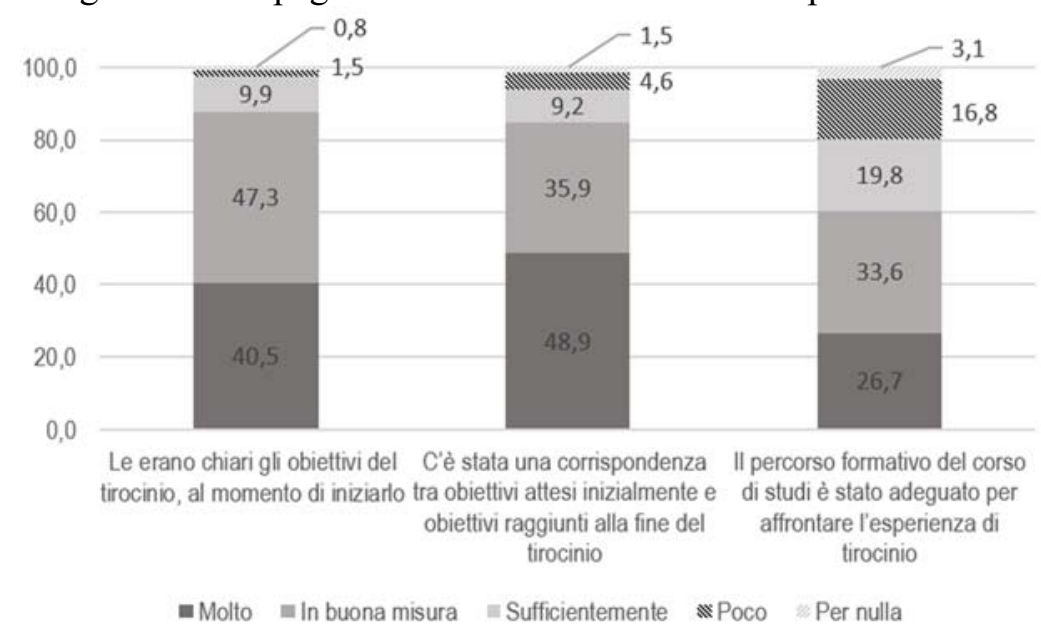

Grafico 1 - Aspettative e constatazioni di sintesi sull'esperienza di tirocinio (domanda 6). Percentuale dei punteggi sul totale delle risposte $(N=131)$

Informazioni interessanti emergono anche dalla comparazione dei risultati delle domande 17 e 18 il cui obiettivo è quello di mettere in relazione la situazione ideale ("quanto ritiene utili i seguenti aspetti al fine di una esperienza di tirocinio ottimale") con la situazione realmente riscontrata ("quanto 
sono stati presenti..."), permettono di comprendere il divario tra le aspettative e la realtà (vedi tabella 1 ).

Tabella 1 - Comparazione tra il valore atteso e quello riscontrato in alcune dimensioni dell'esperienza di tirocinio. Valori percentuali sul totale delle risposte $(N=131)$

\begin{tabular}{lllllll}
\hline Dimensione & Confronto & Molto & $\begin{array}{l}\text { In } \\
\text { buona } \\
\text { misura }\end{array}$ & $\begin{array}{l}\text { Sufficiente- } \\
\text { mente }\end{array}$ & Poco & $\begin{array}{l}\text { Per } \\
\text { nulla }\end{array}$ \\
$\begin{array}{l}\text { Formazione alla cono- } \\
\text { scenza delle dinamiche } \\
\text { operative }\end{array}$ & riscontrato & 37,40 & 33,60 & 13,70 & 9,90 & 5,30 \\
auspicato & 63,36 & 25,95 & 9,16 & 0,76 & 0,76 \\
Preparazione allo svol- & riscontrato & 31,30 & 34,40 & 18,30 & 8,40 & 7,60 \\
Assistenza burocratica & auspicato & 54,96 & 34,35 & 7,63 & 2,29 & 0,76 \\
e amministrativa & riscontrato & 26,70 & 32,80 & 25,20 & 11,50 & 3,80 \\
Accompagnamento & auspicato & 36,64 & 41,22 & 15,27 & 6,87 & 0 \\
alla preparazione & riscontrato & 29,00 & 32,80 & 19,10 & 12,20 & 6,90 \\
\hline
\end{tabular}

Tali dati, se aggregati (vedi grafico 2) evidenziano chiaramente come, nonostante i valori assegnati all'esperienza svolta siano comunque positivi, esiste una distanza tra questi e quelli assegnati a una situazione ideale ${ }^{3}$.

Formazione alla conoscenza delle dinamiche operative
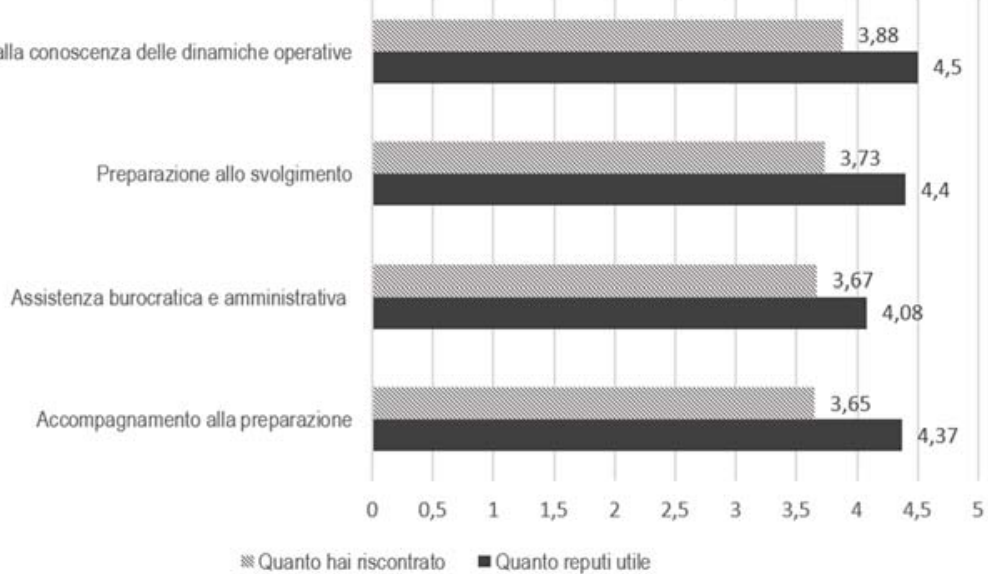

Grafico 2 - Comparazione tra i punteggi medi attribuiti in risposta alle domande 17 e 18

Le risposte aperte contribuiscono a interpretare i dati evidenziando l'esigenza di momenti strutturati dedicati all'orientamento e di incontri con

\footnotetext{
${ }^{3}$ Il calcolo è stato operato moltiplicando le frequenze riscontrate per ognuna delle risposte assegnate alle cinque dimensioni della scala con i seguenti valori: Molto $=5$; In buona misura $=4$; Sufficientemente $=3$; Poco $=2$; Per nulla $=1$ diviso il numero dei rispondenti (131 soggetti). Un valore tra 3 e 4 significa dunque tra "sufficientemente" e "in buona misura", uno tra 4 e 5 tra "in buona misura" e "molto".
} 
esperti del mondo dell'educazione in grado di presentare e orientare rispetto agli aspetti caratterizzanti di tale esperienza. Che i docenti tutor universitari siano ritenuti, in qualche misura meno efficaci dei tutor aziendali, ovvero degli educatori che li hanno accolti nelle strutture, è in parte rilevabile anche dalle risposte alle domande 18.5 e 18.6 che, appunto, richiedevano di indicare la qualità del supporto ricevuto nel corso del tirocinio (grafico 3 ). In termini di valore medio il supporto del tutor universitario viene valutato dai rispondenti, con un punteggio medio sulla scala di 3,8 , mentre il supporto del tutor aziendale $4,25^{4}$. La scelta del Corso di studi di assegnare a tutti i docenti del corso, indipendentemente dall'area di ricerca o dall'insegnamento impartito, le funzioni di docente tutor rappresenta del resto una criticità nota, resa però indispensabile dall'assenza di tutor strutturati o a contratto da dedicare esclusivamente a questa specifica funzione come invece avviene, ad esempio, nei corsi di Scienze della formazione primaria (torneremo sulla questione nella discussione finale).



Grafico 3 - Valutazione del supporto offerto nel corso del tirocinio dal tutor universitario e quello degli enti accoglienti (domande 18.5 e 18.6). Percentuali sul totale risposte $(N=131)$

Nel complessivo, comunque, le esperienze di tirocinio vengono valutate positivamente dal punto di vista formativo e della professionalizzazione. La maggior parte degli studenti ritiene infatti che il tirocinio abbia in buona misura contribuito ad arricchire le proprie conoscenze sia teoriche che metodologiche. Gli esiti delle domande 8 e 10 mostrano come la quasi totalità degli studenti che hanno concluso la loro esperienza di tirocinio riconoscono che

${ }^{4}$ Il calcolo è eseguito con le stesse modalità descritte nella nota 3. 
questo abbia contribuito in modo importante a migliorare ed arricchire la propria formazione (grafico 4). Le due domande chiedevano, infatti, di valutare alcuni descrittori connessi al profilo professionale in via di definizione, chiedendo in quale misura questi fossero presenti prima del tirocinio e in quale misura sono stati constatati dopo.

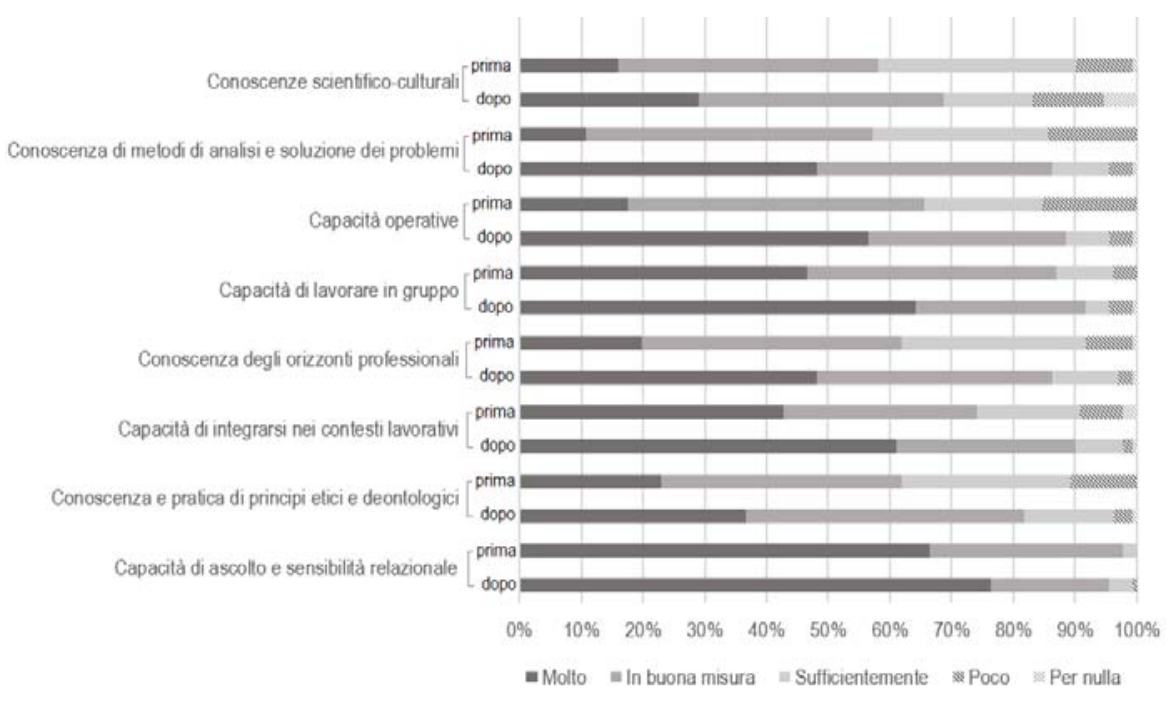

Grafico 4 - Risposte alle domande 8 e 9 sulla valutazione descrittori del profilo professionale prima e dopo il tirocinio. Valori percentuali sul totale delle risposte $(N=131)$

L'incremento avviene su ogni dimensione analizzata, ma le percentuali di incremento dei valori sulle singole dimensioni, se riportate in punteggio medio della scala (vedi tabella 2), mostrano un deciso aumento soprattutto di alcune dimensioni. In particolare, al tirocinio vengono attribuite, come del resto prevedibile, prerogative importanti sul piano dello sviluppo di quelle competenze pratiche che possono esprimersi solo in situazione: conoscenza di metodi di analisi e soluzione problemi (incremento del 21,38\%), capacità operative $(+19,50 \%)$, conoscenze degli orizzonti professionali $(+15,57 \%)$, conoscenza e pratica di principi etici e deontologici $(+10,61 \%)$, capacità di integrarsi nei contesti lavorativi $(+10,55 \%)$. Non si attribuiscono invece al tirocinio particolari capacità di sviluppare quegli aspetti che sono evidentemente già presenti e largamente esperiti nella pratica universitaria come la capacità di ascolto e sensibilità relazionale $(+1,48 \%)$, le conoscenze scientifico-culturali $(+3,36 \%)$ o la capacità di lavorare in gruppo $(+4,8 \%)$.

Confermano il miglioramento in alcune capacità direttamente collegabili al ruolo professionale le risposte date alle domande 12 e 13 (grafico 5). In questo caso elaborando i dati, ovvero trasformando le risposte in punteggi medi delle 
risposte date sulla scala ${ }^{5}$, le percentuali di incremento riscontrate sono in ordine: la capacità progettare (incremento percepito del 16,3\%), gestire $(+16,04 \%)$, realizzare $(+15,42 \%)$, valutare $(+8,52 \%)$ e analizzare $(+7,66 \%)$. Le ultime due dimensioni, valutare e analizzare, sono evidentemente più richieste anche nello studio e se ne attribuisce dunque una minore all'esperienza di tirocinio.

Tabella 2 - Confronto tra i valori riportati in punteggio medio della scala alle risposte date alle domande 8 e 9 sulla valutazione descrittori di professionalità. $(N=131)$

\begin{tabular}{|c|c|c|c|}
\hline Dimensione analizzata & Prima/dopo & Media & Incremento \\
\hline \multirow{2}{*}{ Conoscenze scientifico-culturali } & prima & 3,63 & \multirow{2}{*}{$+3,36 \%$} \\
\hline & dopo & 3,76 & \\
\hline \multirow{2}{*}{$\begin{array}{l}\text { Conoscenza di metodi di analisi e soluzione dei } \\
\text { problemi }\end{array}$} & prima & 3,53 & \multirow{2}{*}{$+21,38 \%$} \\
\hline & dopo & 4,29 & \\
\hline \multirow[t]{2}{*}{ Capacità operative } & prima & 3,68 & \multirow{2}{*}{$+19,50 \%$} \\
\hline & dopo & 4,40 & \\
\hline \multirow[t]{2}{*}{ Capacità di lavorare in gruppo } & prima & 4,30 & \multirow{2}{*}{$+4,80 \%$} \\
\hline & dopo & 4,50 & \\
\hline Conoscenza degli orizzonti professionali & prima & 3,73 & $+15,57 \%$ \\
\hline \multirow{2}{*}{ Capacità di integrarsi nei contesti lavorativi } & prima & 4,05 & \multirow[b]{2}{*}{$+10,55 \%$} \\
\hline & dopo & 4,48 & \\
\hline \multirow{2}{*}{$\begin{array}{l}\text { Conoscenza e pratica di principi etici e deonto- } \\
\text { logici }\end{array}$} & prima & 3,74 & \multirow{2}{*}{$+10,61 \%$} \\
\hline & dopo & 4,14 & \\
\hline \multirow{2}{*}{ Capacità di ascolto e sensibilità relazionale } & prima & 4,64 & \multirow{2}{*}{$+1,48 \%$} \\
\hline & dopo & 4,71 & \\
\hline
\end{tabular}

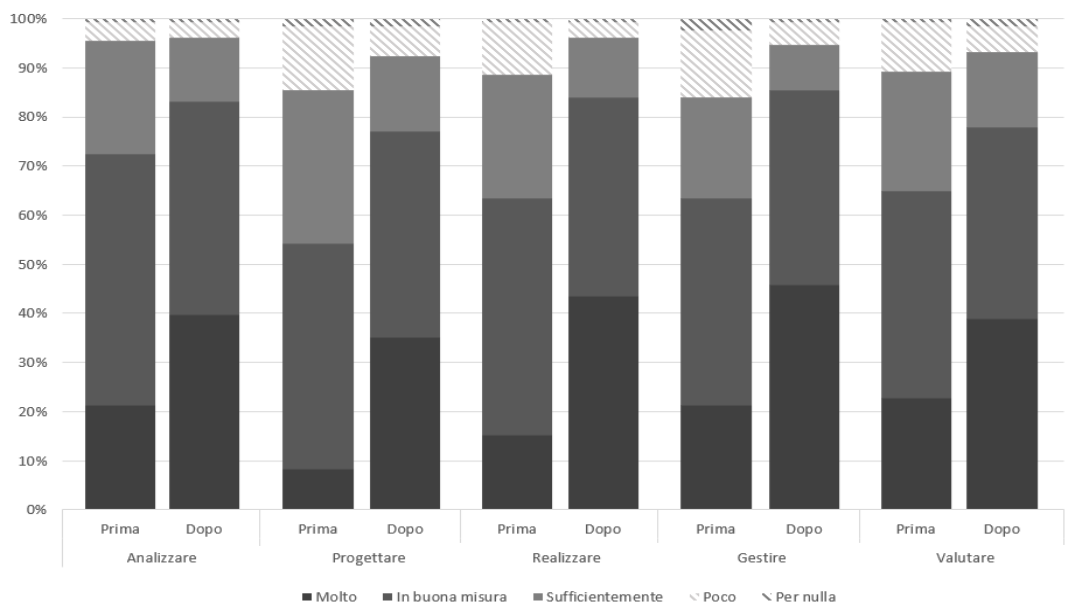

Grafico 5 - Sviluppo di competenze prima e dopo il tirocinio (domande 12 e 13). Valori percentuali sulle singole dimensioni. Analisi sul totale delle risposte $(N=131)$

\footnotetext{
${ }^{5} \mathrm{Il}$ calcolo, anche in questo caso, è eseguito con le stesse modalità descritte nella nota 3.
} 


\section{Le interviste}

Avendo un giudizio complessivo positivo dell'esperienza oggetto d'analisi, ci si è successivamente proposti di approfondire gli aspetti ai quali gli studenti conferiscono un'importanza maggiore e sui quali sentono si possa migliorare ancora. Infatti, alla luce di questi dati si vuole indagare meglio quali siano le motivazioni intrinseche ed estrinseche che li guidano nelle scelte delle strutture presso le quali svolgere l'esperienza e quali sentimenti siano prevalsi durante questo percorso nei vari momenti che lo caratterizzano. Inoltre, è opportuno precisare che, le persone intervistate appartengono ad esperienze svolte in ambiti differenti tra loro. Si spazia dalla comunità per minori alla disabilità, dalla educativa scolastica alla musicoterapia, dallo sport alle dipendenze. Perciò, da questo punto di vista non vi è ripetizione alcuna legata ad ambienti e contesti, ma una eterogeneità che permetterà di raccogliere testimonianze diversificate.

Le interviste one-to-one, della durata di circa 30 minuti ciascuna, sono state realizzate tramite video call sulla piattaforma Teams, a causa delle restrizioni imposte dalla situazione pandemica. I partecipanti a questa sessione di approfondimento sono stati otto studenti che hanno dato la disponibilità. Le domande guida del colloquio sono principalmente tre, ma trattandosi di un'intervista semi strutturata sono state di volta in volta ampliate in base alle risposte e alle singole esperienze. La struttura base comprendeva le seguenti domande:

1. Con quali criteri e sulla base di quale consapevolezza hai scelto l'ente in cui svolgere il tirocinio?

2. Quale è stato il tuo primo approccio con la struttura?

3. L'aspettativa che avevi nei confronti dell'esperienza è stata confermata dalla realtà?

Tutte le domande, soprattutto la seconda e la terza, sono volte ad indagare anche lo stato emotivo che lo studente vive nel corso di questi momenti.

Una condizione che ritorna in ogni esperienza è quella connessa al primo momento del tirocinio, quindi all'esordio dello studente in questo contesto professionalizzante. Ciò che emerge comunemente è la sensazione iniziale di spaesamento, spiazzamento, quello che è stato definito un "impatto forte" all'interno di un mondo nuovo, ma soprattutto volontà di mettersi alla prova in un contesto educativo reale. Nel corso di un'intervista, questo concetto è ben esposto da una studentessa che afferma:

Volevo mettermi alla prova in quell'ambito specifico perché mi incuriosivano i meccanismi che potevano esserci all'interno e come li vivevano gli ospiti[...]. 
Ma anche la consapevolezza dell'importanza professionale di una occasione come quella dei tirocini. E di quanto questo sia cruciale nella crescita del futuro educatore, come evidenzia una studentessa:

L'occasione di tirocinio è una e volevo sfruttarla al meglio svolgendolo dove sarei potuta crescere[...].

La sensazione di spaesamento, spesso, si dissipa nel proseguire dell'esperienza, man mano che si impara a maneggiare gli strumenti della professione. Questi dubbi ed incertezze son però spesso collegate ai sentimenti che caratterizzano la persona che andrà a vivere l'esperienza. Il parere di una studentessa è, da questo punto di vista, molto evocativo:

[...] sono entrata e ho avuto un po' di timore, non posso non dirlo perché è vero lo ho avuto, proprio un po' di paura di non riuscire ad essere all'altezza, in grado di fare quello che avrei dovuto fare e quindi anche tramite l'osservazione [...] imparavo a muovermi. Non è stato un ingresso sereno perché è comunque una responsabilità, non stiamo andando ad osservare cosa accade in modo neutro[...].

Emozioni contrastanti che portano gli studenti a mettere in discussione le proprie capacità per poi scoprire come la pratica in sé ha dato loro le certezze delle quali avevano bisogno. A riguardo, un'affermazione particolarmente illuminante:

[...] È stato determinante sotto tutti i punti di vista, soprattutto quello motivazionale.

In tutte le esperienze raccolte la figura del tutor aziendale appare determinante. Il suo accompagnamento è cruciale nel consolidamento delle competenze necessarie dell'agire educativo, ragion per cui il suo ruolo di collegamento tra teoria e pratica si rivela decisivo ed essenziale. Lo conferma la testimonianza di uno studente, che afferma:

[...] Sapevo cosa avrei dovuto affrontare, mi era stato spiegato chiaramente, quindi ero consapevole del ruolo che avrei avuto e delle azioni che avrei dovuto svolgere [...] chiaramente ho avuto dei momenti di difficoltà all'interno di situazioni specifiche mai affrontate [...] ma son riuscito a gestire anche queste situazioni perché mi son sentito accompagnato, nel percorso che ho fatto, dalle figure di riferimento.

In alcuni contesti queste figure si impensieriscono riguardo la posizione del tirocinante all'interno della struttura, cercando di prepararli il più possibile al contesto. Questo, a volte, può sfociare in un sentimento di protezione 
esagerato che impedisce allo studente di avere un contatto diretto con le persone con le quali si andranno a confrontare. Ma anche in situazioni così particolari, il dialogo e confronto sincero tra tutor e tirocinante dimostra essere la strada giusta verso il superamento di questi timori, come dimostra l'esperienza di una studentessa:

[...] inizialmente sono stata "relegata" all'interno di uno degli studi per informarmi sui vari temi e situazioni legate a questo mondo. Un mese l'ho passato andando in struttura a studiare. Questo lo facevano per tutelarmi dalla situazione difficile che c'era all'interno, ma io volevo affrontarla questa realtà perché son andata apposta, non per continuare a studiare [...]. Poi, nel momento in cui mi sarei resa conto che non sarei stata in grado avrei fatto io stessa un passo indietro, ma così non è stato. Non pretendevo di svolgere compiti oltre le mie responsabilità [...] ma capirli, mettermi al loro pari, provare a comprendere la base dei loro ragionamenti che poi hanno portato a determinate azioni. Perciò ne ho parlato apertamente [...] e una volta capita la mia motivazione mi ha permesso di svolgere le attività educative che mi competevano[...].

Ancora una volta la voglia di mettersi in gioco e alla prova insita nel tirocinante emerge con forza e si fa notare finché non ottiene ciò che gli spetta: l'esperienza professionale necessaria alla preparazione di un mestiere. Quell'avventura che permette ai futuri educatori di maturare ancor di più e aggiungere un altro tassello alla loro crescita professionale, che non corrisponde al possedere tutti gli strumenti utili allo svolgimento della pratica educativa, bensì all'avere consapevolezza della ricostruzione continua che caratterizza il lavoro dell'educatore. Questo nodo fondamentale è ben descritto da una delle studentesse intervistate, che dice:

[...] Sarai sempre improvvisato, la laurea non ti garantisce il saper fare, poi cresci fiorisci, fai esperienze. Ogni giorno è nuovo ed è anche meglio che sia così. Ogni giorno una scoperta perché per me, e penso per il lavoro di un qualunque educatore e pedagogista, sia il riformulare, il ricalcolare ogni giorno una partenza continua. [...] non dare per scontato ecco, per me è questo. E credo che questo sia l'insegnamento che mi son portata via dal tirocinio, come da ogni esperienza lavorativa, perché se dai per scontato il tuo procedere è finita.

\section{Considerazioni conclusive}

I dati emersi dalle due rilevazioni, il questionario e le interviste, hanno permesso alla Commissione tirocini del Corso di studi in Scienze dell'edu- 
cazione e della formazione dell'Università degli Studi di Cagliari di comprendere meglio i punti di forza e di debolezza delle esperienze di tirocinio. Tali acquisizioni hanno permesso, grazie anche alle occasioni di incontro e confronto con le esperienze degli altri atenei appartenenti alla rete del Progetto POT "Percorsi di orientamento e tutorato per promuovere il successo universitario e professionale", di assumere una serie di decisioni tese sia a facilitare l'orientamento e l'accompagnamento degli studenti in particolare ai percorsi professionalizzazione, sia al monitoraggio degli esiti.

Il problema di riuscire a fornire una guida iniziale efficace si scontra con la mancanza di una figura stabile, adeguatamente preparata e dedicata in maniera esclusiva a tale scopo. Il Corsi di studi dell'area pedagogica non beneficiano di figure istituzionalmente previste allo scopo così come avviene, ad esempio, nel Corso di Scienze della formazione primaria dove ricordiamo sono presenti le figure del tutor organizzatore e quella del tutor coordinatore, entrambi docenti (o dirigenti) in servizio presso la scuola primaria o dell'infanzia comandati presso le università al fine di accompagnare gli studenti nei loro tirocini e assicurare, al contempo, il raccordo con le scuole. Nel panorama italiano i corsi della classe L-19 hanno provato ad affrontare il problema, che rappresenta una vera e propria lacuna normativa, in modi diversi. All'Università di Cagliari, da anni, il ruolo del tutor interno viene svolto dai singoli docenti del Corso di studi che ricevono uno stesso numero di studenti suddivisi alfabeticamente sulla base delle iniziali del cognome. Questa soluzione, se da un lato ha il vantaggio di un'equa ripartizione del carico di lavoro tra i docenti, presenta anche - come intuibile - numerosi problemi e limitazioni. Innanzi tutto, i docenti di aree disciplinari meno prossime all'ambito pedagogico hanno una minore familiarità con $i$ contesti professionali e le tematiche a questi connesse. Inoltre, e per tutti, la gestione di un'attività come questa che richiederebbe tempo e dedizione continui (tenendo anche conto dell'elevato numero di studenti) che mal si conciliano con i numerosi impegni che ogni docente ha.

Da qui la ricerca continua di soluzioni in grado di alleggerire i docenti da questo incarico e di integrare e uniformare il tipo di supporto offerto agli studenti. Gli esiti delle indagini e delle riflessioni conseguenti hanno portato a prendere due diverse decisioni. Da una parte quella di allestire un ambiente online, arricchito da materiale audiovisivo e documentario finalizzato al primo orientamento, dall'altra quella di inserire un ulteriore laboratorio didattico obbligatorio denominato "Laboratorio di orientamento al tirocinio curriculare", della durata di 25 ore con il conseguimento di 1 credito formativo.

Ciò risponderà alle necessità emerse dalla conduzione delle interviste. Prima tra tutte, quella di eliminare la sensazione di spaesamento ed impreparazione che gli studenti vivono prima di iniziare l'esperienza. L'attivazione 
di un laboratorio propedeutico all'esperienza consentirà di fornire quell'orientamento e quel supporto informativo necessari per affrontare il tirocinio nei suoi diversi aspetti: normativo, metodologico, documentario, deontologico e formativo.

Gli argomenti sollevati dagli studenti imponevano di intraprendere una decisione importante sul fronte dell'individuazione di una figura stabile per lo studente tirocinante, una figura capace di accompagnarlo nell'avvio del percorso, quindi nell'orientamento, ma anche attraverso l'osservazione delle fasi del percorso e la riflessività che caratterizza il momento finale dell'esperienza. Il laboratorio non solo sarà introduttivo al tirocinio ma soprattutto propedeutico al suo avvio dal punto di vista burocratico: non sarà possibile iniziare il percorso senza averlo frequentato.

Un ulteriore aspetto su cui il Corso ha lavorato al fine di rendere il percorso di tirocinio ancor più completo e preparare meglio gli studenti all'incontro con le realtà lavorative, è stato perfezionato anche sotto i suggerimenti forniti dagli altri Corsi di studio aderenti alla rete del progetto POT. In particolare, l'implementazione di uno spazio all'interno di un ambiente online, all'interno del quale offrire un vero e proprio percorso di preparazione ed accompagnamento al tirocinio con una varietà di risorse e materiali di lavoro (vademecum, documenti, letture e bibliografie) oltre a una serie di risorse audiovisivi (tutoriali, video interviste, testimonianze e brevi documentari) tese a mostrare la varietà dei servizi, delle strutture e delle reti territoriali, le specificità dei contesti in cui l'educatore e il pedagogista si trovano ad operare, l'identità professionale, le responsabilità e le modalità di intervento. L'allestimento di questo spazio, che è diventato particolarmente utile nel periodo in cui, a causa del lockdown decretato nel periodo della massima incidenza della pandemia da COVID-19, è stato necessario attivare tirocini online sostitutivi di quelli presso gli enti esterni (esperienze riservate esclusivamente ai laureandi), risponde alla necessità emersa dal questionario di fornire un sostegno stabile capace di guidare maggiormente nei momenti preliminari dell'esperienza di tirocinio come in quelli conclusivi legati alla stesura della relazione finale.

L'ascolto degli studenti, lo scambio di idee e di opinioni con le loro rappresentanze, ha permesso di far affiorare punti di vista e suggerimenti che sarebbero stati difficilmente individuabili. Questi primi correttivi dovranno naturalmente essere valutati sul piano dell'effettiva capacità di miglioramento ed è plausibile che saranno necessarie ulteriori azioni, ma non c'è dubbio che sul piano operativo mettere al centro gli studenti abbia contribuito all'assunzione di uno sguardo più ampio oltre ad aver aumentato il senso di coesione e di appartenenza in quanti, studenti compresi, hanno collaborato a questo lavoro. 


\section{Bibliografia}

Bastianoni, P., \& Spaggiari, E. (2016). Il ruolo del tirocinio nella formazione dell'educatore. Annali Online Della Didattica e Della Formazione Docente, 8(11), pp. 69-84. Doi: 10.15160/2038-1034/1160.

Bauman, Z. (2008). Vita liquida. Roma-Bari: Editori Laterza.

Bufalino, G. (2000). Il ruolo del tirocinio formativo nella costruzione del futuro professionista, Formazione, Lavoro, Persona, 9, pp. 45-53.

Cook-Sather, A. (2002). Authorizing Students' Perspectives: Toward Trust, Dialogue, and Change in Education. Educational Researcher, 31(4), pp. 3-14. Retrieved from https://doi.org/10.3102/0013189X031004003.

De Canale, B. (2015). Il tirocinio formativo: catalizzatore di competenza. Formazione, Lavoro, Persona, 5(15), pp. 71-83.

Deiana, S. (2019). Pedagogisti e pedagogiste tra formazione e lavoro. Narrazioni e letture della scena pedagogica universitaria e professionale. Lecce: Pensa Multimedia.

Del Gobbo, G., Frison, D., \& Pellegrini, M. (2021). Verso un modello di self-directed career management: una proposta di tirocinio digitale. Formazione, Lavoro, Persona, 9(33), pp. 149-170.

Dewey, J. (1961). Come pensiamo. Firenze: La Nuova Italia.

Di Vita, A. (2017). «Student Voice» per la valorizzazione delle risorse personali. Journal of Educational, Cultural and Psychological Studies, (16), pp. 269-294. Retrieved from https://doi.org/10.7358/ecps-2017-016-divi.

Fabbri, L. (2018). La costruzione dei contenuti core come pratica scientifica condivisa. Metodologie per la trasformazione della progettazione dell'offerta formativa. Form@re - Open Journal per La Formazione in Rete, 18(3), pp. 61-69. Doi: 10.13128/formare-24614.

Fielding, M. (2001). Beyond the Rhetoric of Student Voice: new departures or new constraints in the transformation of 21st century schooling?. Forum, 43(2), 100. Retrieved from https://doi.org/10.2304/forum.2001.43.2.1.

Grion, V., \& Cook-Sather, A. (2013). Student Voice. Prospettive internazionali e pratiche emergenti in Italia. Milano: Guerini

Lave, J., \& Wenger, E. (2007). L'apprendimento situato. Dall'osservazione alla partecipazione attiva nei contesti sociali. Trento: Erickson.

Mezirow, J. (2003). Apprendimento e trasformazione. Il significato dell'esperienza e il valore della riflessione nell'apprendimento degli adulti. Milano: Raffaello Cortina.

Mortari, L. (2009). Ricercare e riflettere. La formazione del docente professionista. Roma: Carocci.

Santoro, D. (2015) Il tirocinio curricolare in tempi di crisi: una leva strategica di placement. Formazione, Lavoro, Persona, 15(5), pp. 129-134.

Schön, D. A. (1993). Il professionista riflessivo. Per una nuova epistemologia della pratica professionale. Bari: Dedalo. 
Spuznar, G., \& Renda, E. (1998). Educatori non si nasce. Una riflessione sul ruolo del tirocinio nella formazione delle competenze per il lavoro socio-educativo. Formazione, Lavoro, Persona, 15, pp. 149-159.

Tramma, S. (2008). Dentro e fuori la scuola. Milano: Mondadori

Wenger, E. (2006). Comunità di Pratica. Apprendimento, significato e identità. Milano: Raffaello Cortina. 
Allegato 1. Il questionario

1. Nome

2. Cognome

3. Matricola

4. Corso di studi

5. Quale tirocinio hai svolto?

6. Aspettative e prime constatazioni. Scegliere la risposta appropriata per ciascun elemento:

\begin{tabular}{|c|c|c|c|c|c|}
\hline (5 = molto, $4=$ in buona misura, $3=$ sufficientemente, $2=$ poco, $1=$ per nulla) & 5 & 4 & 3 & 2 & 1 \\
\hline 1. Le erano chiari gli obiettivi del tirocinio, al momento di iniziarlo & & & & & \\
\hline $\begin{array}{l}\text { 2. C'è stata una corrispondenza tra obiettivi attesi inizialmente e obiettivi raggiunti alla } \\
\text { fine del tirocinio }\end{array}$ & & & & & \\
\hline $\begin{array}{l}\text { 3. Il percorso formativo del corso di studi è stato adeguato per affrontare l'esperienza } \\
\text { di tirocinio }\end{array}$ & & & & & \\
\hline
\end{tabular}

di tirocinio

7. Osservazioni elo suggerimenti:

8. Di seguito sono riportati alcuni descrittori del profilo professionale che sta andando a conseguire. Quanto ritiene che ognuno di questi la caratterizzasse prima di cominciare il tirocinio?

\begin{tabular}{|c|c|c|c|c|c|}
\hline ( 5 = molto, 4 = in buona misura, 3 = sufficientemente, $2=$ poco, 1 = per nulla) & 5 & 4 & 3 & 2 & 1 \\
\hline 1. Conoscenze scientifico-culturali & & & & & \\
\hline 2. Conoscenza di metodi di analisi e soluzione dei problemi & & & & & \\
\hline 3. Capacità operative & & & & & \\
\hline 4. Capacità di lavorare in gruppo & & & & & \\
\hline 5. Conoscenza degli orizzonti professionali & & & & & \\
\hline 6. Capacità di integrarsi nei contesti lavorativi & & & & & \\
\hline 7. Conoscenza e pratica di principi etici e deontologici & & & & & \\
\hline 8. Capacità di ascolto e sensibilità relazionale & & & & & \\
\hline
\end{tabular}

9. Osservazioni elo suggerimenti:

10. In quale misura ritiene che il tirocinio abbia contributo a migliorare la sua professionalità in relazione ai seguenti descrittori?

\begin{tabular}{|c|c|c|c|c|c|}
\hline ( $5=$ molto, 4 = in buona misura, $3=$ sufficientemente, $2=$ poco, 1 = per nulla) & 5 & 4 & 3 & 2 & 1 \\
\hline \multicolumn{6}{|l|}{ Conoscenze di saperi scientifico-culturali } \\
\hline \multicolumn{6}{|l|}{ Conoscenza di metodi di analisi e soluzione dei problemi } \\
\hline \multicolumn{6}{|l|}{ Capacità operative } \\
\hline \multicolumn{6}{|l|}{ Capacità di lavorare in gruppo } \\
\hline \multicolumn{6}{|l|}{ Conoscenza degli orizzonti professionali } \\
\hline \multicolumn{6}{|l|}{ Capacità di integrarsi nei contesti lavorativi } \\
\hline \multicolumn{6}{|l|}{ Conoscenza e pratica di principi etici e deontologici } \\
\hline Capacità di ascolto e sensibilità relazionale & & & & & \\
\hline
\end{tabular}

11. Osservazioni elo suggerimenti:

12. In quale misura ritiene, nella sua formazione universitaria, di aver acquisito le seguenti capacità connesse alla lettura dei mutamenti e delle esigenze educative e al trasformare ciò in interventi, processi e servizi nell'ambito della formazione e dell'educazione:

\begin{tabular}{|l|l|l|l|l|l|}
\hline (5= molto, 4 = in buona misura, 3 = sufficientemente, 2 = poco, 1 = per nulla) & 5 & 4 & 3 & 2 & 1 \\
\hline 1. Analizzare & & & & & \\
\hline 2. Progettare & & & & & \\
\hline 3. Realizzare & & & & & \\
\hline 4. Gestire & & & & & \\
\hline 5. Valutare & & & & & \\
\hline
\end{tabular}


13. In quale misura ritiene che il tirocinio abbia contribuito a migliorare la sua professionalità nelle seguenti capacità connesse alla lettura dei mutamenti e delle esigenze educative e al trasformare ciò in interventi, processi e servizi nell'ambito della formazione e dell'educazione:

\begin{tabular}{|c|c|c|c|c|c|}
\hline ( $5=$ molto, 4 = in buona misura, $3=$ sufficientemente, $2=$ poco, 1 = per nulla) & 5 & 4 & 3 & 2 & 1 \\
\hline 1. Analizzare & & & & & \\
\hline 2. Progettare & & & & & \\
\hline 3. Realizzare & & & & & \\
\hline 4. Gestire & & & & & \\
\hline 5. Valutare & & & & & \\
\hline
\end{tabular}

14. Quale canale ha utilizzato per scegliere l'entella struttura in cui ha svolto il tirocinio?

- consultazione bacheca d'Ateneo/ufficio placement e tirocini

- conoscenze personali

- contatti con professori e/o tutor universitari

- candidatura spontanea

- Altro

15. In base a quali criteri ha scelto l'ente/la struttura in cui svolgere il tirocinio?

- $\quad$ Aspetti operativi-logistici (tempistica breve, vicinanza territoriale della sede, ecc.)

- L'ambito di attività e gli specifici destinatari della relazione educativa

- In quanto migliore alternativa disponibile rispetto ad una scelta preferenziale apparsa impraticabile

- Altro

16. Prima e durante lo svolgimento del tirocinio, si è documentato e ha ricercato ulteriori approfondimenti sui saperi, i contesti, il ruolo e le pratiche dell'educatore, con particolare attenzione a ciò che poteva connettersi alla sua specifica esperienza di tirocinio?

- Sì

- In parte

- No

17. Valuti quanto ritiene utili i seguenti aspetti al fine di una esperienza di tirocinio ottimale:

\begin{tabular}{|c|c|c|c|c|c|}
\hline (5 = molto, $4=$ in buona misura, $3=$ sufficientemente, $2=$ poco, $1=$ per nulla) & 5 & 4 & 3 & 2 & 1 \\
\hline 1. Accompagnamento alla preparazione del tirocinio sul piano progettuale & & & & & \\
\hline 2. Assistenza burocratica e amministrativa & & & & & \\
\hline $\begin{array}{l}\text { 3. Preparazione allo svolgimento (riflessioni sulle motivazioni, conoscenza delle figure } \\
\text { professionali e dei contesti operativi) }\end{array}$ & & & & & \\
\hline $\begin{array}{l}\text { 4. Formazione alla conoscenza delle dinamiche operative, comunicative e relazionali } \\
\text { nei contesti di lavoro }\end{array}$ & & & & & \\
\hline
\end{tabular}

18. Valuti quanto sono stati presenti nella sua esperienza di tirocinio i seguenti aspetti:

\begin{tabular}{|c|c|c|c|c|c|}
\hline ( $5=$ molto, $4=$ in buona misura, $3=$ sufficientemente, $2=$ poco, $1=$ per nulla) & 5 & 4 & 3 & 2 & 1 \\
\hline 1. Accompagnamento alla preparazione del tirocinio sul piano progettuale & & & & & \\
\hline 2. Assistenza burocratica e amministrativa & & & & & \\
\hline $\begin{array}{l}\text { 3. Preparazione allo svolgimento (riflessioni sulle motivazioni, conoscenza delle figure } \\
\text { professionali e dei contesti operativi) }\end{array}$ & & & & & \\
\hline $\begin{array}{l}\text { 4. Formazione alla conoscenza delle dinamiche operative, comunicative e relazionali } \\
\text { nei contesti di lavoro }\end{array}$ & & & & & \\
\hline 5. Supporto complessivo da parte del tutor universitario & & & & & \\
\hline 6. Supporto complessivo da parte del tutor aziendale & & & & & \\
\hline
\end{tabular}

19. Osservazioni elo suggerimenti:

20. Al termine del tirocinio, le è stata fatta una proposta di inserimento nell'ente presso cui lo ha svolto?

- Sì, e ho accettato la proposta di inserimento

- Sì, ma non ho accettato la proposta di inserimento

- No, non mi è stata fatta alcuna proposta 
21. Nel complesso, come giudica la sua esperienza di tirocinio?

- Molto positiva

- Abbastanza positiva

- Sufficiente

- Abbastanza negativa

- Molto negativa

22. Osservazioni elo suggerimenti: 